\title{
GLCM of Fuzzy Clustering Means for Textural Future Extraction of Brain Tumor in Probabilistic Neural Networks
}

\author{
Shaik Salma Begum, D.Rajya Lakshmi
}

\begin{abstract}
Brain tumor is one of the major causes of death among people. It is evident that the chances of survival can be increased if the tumor is detected and classified correctly at its early stage. normal strategies encompass obvious strategies, as an instance, biopsy, lumbar reduce and spinal faucet technique, to distinguish and installation thoughts tumors into generous (non unstable) and threatening (adverse). A pc supported give up calculation has been planned in order to build the precision of mind tumor region and grouping, and along the ones strains supplant traditional intrusive and tedious techniques. This paper offers a powerful technique for mind tumor grouping, wherein, the actual Magnetic Resonance (MR) snap shots are prepared into ordinary, non risky (thoughtful) cerebrum tumor and damaging (risky) cerebrum tumor. The proposed approach to the pursuit of three levels: (1) wavelet damage, (2) the texture extraction spotlight and (three) order. Discrete remodel Wavelet first achieved by using Daubechies wavelet (DB4), to deteriorate the MR image to various ranges loud and nitty coefficient of sand and the stage time darkness co-event lattice general, of which the insight of land, for example, electricity, differentiate, dating, homogeneity and entropy gain. Event co-grid results are then pushed right into probabilistic neural system for each order and identity of the tumor. The proposed technique has been achieved in the MR image is authentic, and the accuracy of clustering using probabilistic neural system is seen as roughly $100 \%$.
\end{abstract}

\section{INTRODUCTION}

Tumor is due to the uncontrolled growth of the tissues in any part of the body. The tumor may be primary or secondary. If the part of the tumor is spread to another place and grown as its own then it is known as secondary (Dhanalakshmi and Kanimozhi, 2013). Tumor is because of the out of manage improvement of the tissues in any piece of the body. The tumor is probably essential or auxiliary. inside the occasion that it is a birthplace, at that issue it's miles called vital. at the off danger that the piece of the tumor is unfold to a few other region and advanced as its very very very own then it's far referred to as optional (Kabade and Gaikwad, 2013). Cerebrum tumor is a assembly of unusual cells that turns into within the thoughts or across the thoughts. Tumors can legitimately annihilate all robust synapses. it may likewise in a roundabout way damage sound cells via swarming one-of-a-kind portions of the mind and inflicting infection, cerebrum growing and pressure within the skull (Logeswari and Karnan, 2010). absolutely programmed conventional and inflamed human thoughts order from Magnetic Resonance pictures (MRI) is critical for studies and clinical examinations. in the ongoing

Revised Manuscript Received on 14, October 2019. Science and Engineering, Jawaharlal Nehru Technology University, Kakinada, A. P. India.

D.Rajya Lakshmi, Principal, University College of Engineering, JNTUK, Narasaraopet, Guntur, A.P, India
Shaik Salma Begum, Research-Scholar, Department of Computer

beyond, the improvement of laptop Aided prognosis (CAD) frameworks for supporting the docs for selecting better options have been the area of intrigue (Rajalakshmi and LakshmiPrabha, 2013).

In image processing, images convey the information where input image is processed to get output also an image. In today's world, the images used are in digital format.. As of late, the presentation of statistics innovation and e-social insurance framework in recuperation vicinity encourages clinical experts to provide better medicinal services to sufferers. This research uncovers the problem department of odd and ordinary tissues from MRI photos using darkish stage co-occasion framework (GLCM) spotlight extraction and probabilistic neural device (PNN) classifier. Brain tumors are rarely out of the development of tissue manipulating negatives in mind. A tumor may experience beneficial and harmful. Liver tumors have the consistency and structure composed of cells of non-dynamic disease. dangerous tumors have non-consistency and structure including malignant cells dynamic boom spread across components. As shown by the mutual aid from around the world are becoming associations, scales evaluating framework implemented from class I to IV testing. evaluation of a wide arrange friendly and threatening tumor. Evaluation I and II is a tumor of low-level ratings, while class III and IV tumors broad level assessment. mind tumor can affect people at any age.

The impact on every individual may not be same. Due to such a complex structure of human brain, a diagnosis of tumor area in brain is challenging task. The malignant-type grade III and IV of tumor is fast growing.. impacts the sound synapses and may unfold to great pieces of the mind or spinal string and is grade by grade dangerous and might live untreated. So reputation of such cerebrum tumor location, identification and affiliation in earlier stage is a top trouble in restorative technology. by using way of enhancing the cutting-edge imaging strategies, it encourages the experts to take a look at and song the event and development of tumorstimulated locales at various stages on the way to take provide less expensive assessment those photographs filtering. The vital issue problem modified into area of mind tumor in starting periods with the aim that legitimate treatment can be received. In view of this information, the maximum appropriate remedy, radiation, medical procedure or chemotherapy may be selected. eventually, it's miles apparent that the possibilities of patience of a tumorinflamed affected person may be multiplied altogether if the

Published By:

Blue Eyes Intelligence Engineering 


\section{GLCM of Fuzzy Clustering Means for Textural Future Extraction of Brain Tumor in Probabilistic Neural Networks}

tumor is recognized precisely in its beginning time. The division have become applied to decide the stimulated tumor element the usage of imaging modalities. branch is way of isolating the photograph to its constituent components sharing indistinguishable homes, for instance, shading, floor, complexity and obstacles.

Analyzing and processing of MRI brain tumor images are the most challenging and upcoming field. Magnetic resonance imaging (MRI) is an advanced medical imaging technique used to produce high-quality frame and it's miles first-rate approach for deciding on the proper remedy at right set up for tumor-infected individual. severa techniques had been proposed for characterization of cerebrum tumors in MR photographs, as an instance, fuzzy clustering means (FCM), support vector machine (SVM), artificial neural network (ANN), knowledge-based techniques, and expectation-maximization (EM) calculation technique which are a part of the well-known strategies implemented for regionbased division consequently to extricate the substantial facts from the medicinal imaging modalities.

John (2012) has introduced an efficient method of brain tumor classification, where, the real Magnetic Resonance (MR)) pix have been grouped into typical, non risky (amiable) thoughts tumor and negative (threatening) mind tumor. Their proposed approach pursues 3 degrees:

- Wavelet decomposition

- Textural feature extraction

- Classification

\section{II.LITERATURE SURVEY}

Bahadure et al. proposed BWT and SVM techniques image analysis for MRI-based brain tumor detection and classification. In this method, accuracy of $95 \%$ was achieved using skull stripping which eliminated all nonbrain tissues for the detection purpose [1]. Joseph et al. [2] Proposed department MRI images utilizing Kmeans experience bunching together of calculation set aside for local tumor morphology photos. Mechanical brain tumor association of MRI images using vector machine bolsters change proposed by the way how Alfonse and Salem [3]. The accuracy of the classifier is emerging as a sophisticated use of the short Fourier changes for extraction of maximum approach highlights and extra significant importance be done to decrease the highlights. Accuracy obtained from the proposed work is $98 \%$. MRI brain images. One part of the district consists of anomalies tumor cells, despite the fact that the following venues including regular synapses [4]. For tumor department mind, Zanaty [5] proposed techniques rely on half of the breed type, with aggregate to grow the seeds, FCM, and Jaccard coefficient calculation and evaluation of the percentage of white dim count cut tissue tumors various number of shots a snap.

The average score of $90 \% \mathrm{~S}$ segmentation is achieved with a noise level of 9-3\%. To manage and protocol address different images and non-linear data grouping real possibility depending on the difference of advanced MRI images, Yao et al. [6] proposed a closed machine surface extraction wavelet and SVM highlighting changes with precision eighty $3 \%$. For branch grouping and tumor mind, Kumar and Vijayakumar [7] proposed the use of a tool principal component analysis (PCA) and the spiral artwork premise of a piece with SVM. They acquire ninety $4 \%$ accuracy with this technique. A nerve fake peripheral devices for each classifier and departments ended affiliates may be utilized to mind tumor from MRI images turn out to be proposed through the use of Sharma et al. [8] using the raw texture finishes highlighting the precision of one hundred\%. For the image department of medicine, a hairy bunching limited by spatial record extraction turned into proposed by Cui et al. [9].

The author used Jaccard similarity index as a measure of segmentation claiming an accuracy of $83-95 \%$ and differentiating in to white, gray and cerebrospinal fluid.. For the mind tumor photograph branch, dynamic form technique became completed to take care of the issue depending on power homogeneities on MRI pictures become proposed via using Wang et al. [10]. For the extraction programmed from highlights and tumor identity with the highlight of the upgrade using a version of aggregate Gaussian performed on pictures MRI with highlights wavelet and the study of the head modified to be proposed by the resources of Chaddad [11] with precision T1-weighted Five Nine\% and T2weighted $90 \%$ to talent shots weighted MRI heartbeat.

\section{III.PROBABILISTIC NEURAL NETWORK (PNN)}

In early 1990s, D.F Specht introduced feed-forward neural network named as probabilistic neural network (PNN). It is derived from Bayesian networks and statistical algorithm called Kernel Fisher discriminant analysis .. it is a mile or hub made of four layers: the input layer, a hidden layer, layer and layer formats the results. PNN plans are friends weighted as nerve gadget. Layer comprised of information from the ' $\mathrm{P}$ ' is no challenge for all of these neurons exit highlights different factors eliminated the use of dim diploma co-chance grating (GLCM). Information stored hundreds hub 1 , and they abilties was passed into the layer of disguise. In the example of the layers, the premise Radial capacity has been determined and has been maintained to a summation layer. Layer consists of the estimated weighted summation initiation in every elegance observed in the hidden layer. Estimates summation layer is maintained to produce the coating. Results of alternate layers noteworthy maximum probability, one incredible show for the purpose of zero indicates the type of beauty and terrible for non-centered kind of grandeur.

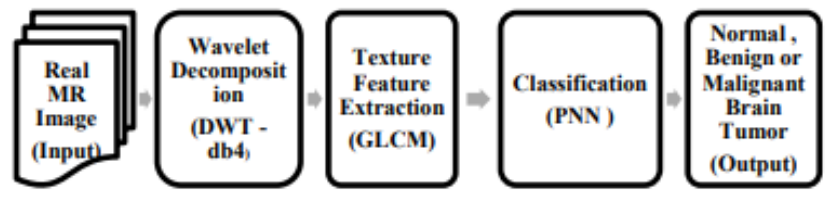

Fig 1.Brain Tumor Classification Process 


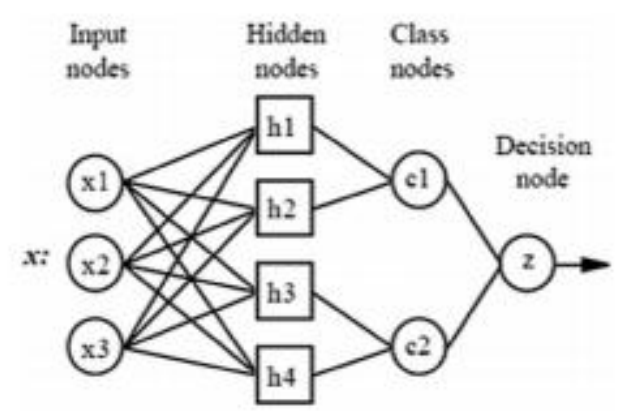

Fig. 2 Diagram of probabilistic neural networks

\section{IV.PROPOSED METHOD FOR DETECTING BRAIN TUMOR}

\subsection{Discrete Wavelet Decomposition}

Wavelets are mathematical powerful tool for feature extraction, and has been used to extract the wavelet coefficients of the MR image. Discrete Wavelet exchange is the use of WT utilize dyadic scale and position. Rules important aspect of DWT offered as pursuit: Let $\mathrm{x}(\mathrm{t})$ is a square function of integrating the whole, then continuously WT of $x(t)$ relative to a given wavelet $\Psi(\mathrm{t})$ is defined as

$$
W_{\Psi(a, b)}=\int_{-\infty}^{\infty} x(t) \Psi_{a, b}(t) d t
$$

Where, the wavelet is calculated from the mother wavelet by expanding and interpretation factor a and b separately, the original positive number.

$$
\Psi_{a, b}(t)=\frac{i}{\sqrt{a}} \Psi\left(\frac{t-a}{b}\right)
$$

Equation (1) can be discretized by holding $\mathrm{A}$ and $\mathrm{B}$ into discrete lattice $(\mathrm{a}=2 \mathrm{~b}$ and $>0$ ) to provide discrete wavelet transform, which can be expressed as,

$$
\begin{aligned}
& a_{j, k}(n)=D S\left[\sum_{n} x(n) g_{j}^{*}\left(n-2^{j} k\right)\right] \\
& d_{j, k}(n)=D S\left[\sum_{n} x(n) g_{j}^{*}\left(n-2^{j} k\right)\right]
\end{aligned}
$$
components and detailed estimates, respectively. The function $\mathrm{g}(\mathrm{n})$ and $\mathrm{h}(\mathrm{n})$ implies lowpass coefficients and moderate-pass channel, one by one. Subscript $\mathrm{j}$ and ok to talk with wavelet scale and interpretation elements, for my part. DS administrator applied for down try. twodimensional DWT carrying around four sub companies LL (lowlow), LH (low-high), HL (high-low), HH (high-high) at each scale. Sub band LL, is the estimation a part of the photo, that is applied for next dimensional DWT. at the same time as, LH, HL, HH are the factor with the resource of the usage of factor segments of the photograph alongside the flat, vertical and nook to corner hub, one after the opposite, as appeared inside the determine three.

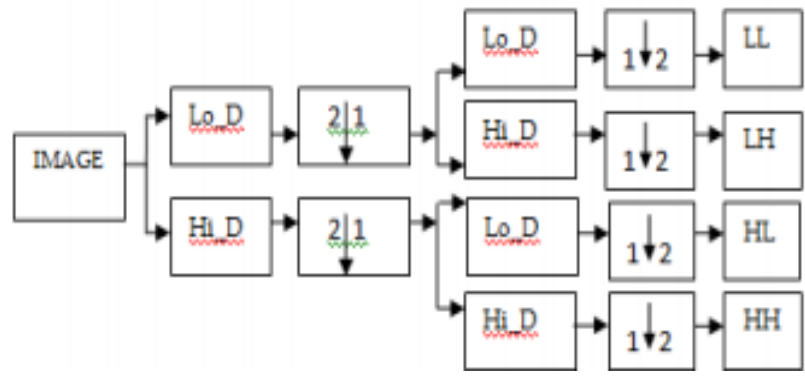

Fig. 3. 2D Discrete Wavelet Transform
Where, the coefficients $a_{j, k}$ and $d_{j, k}$ refers to the

\section{Lo_D - Low Pass Filter}

Ho_D - High Pass Filter

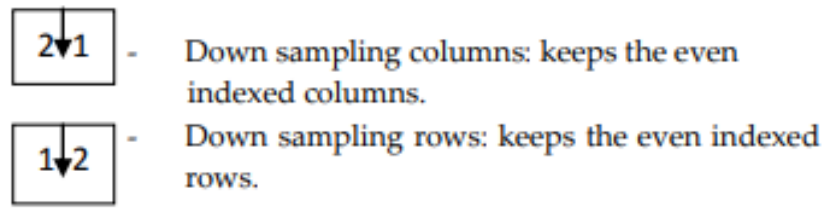

In view of the writing look at, Daubechies wavelet is considered due to the fact the nice amongst particular wavelets for photo software software and LH, HL subbusinesses had better contrasted with the highlights from LL sub band. Henceforth on this technique, a five diploma deterioration utilising daubechies wavelet have end up registered and the highlights were extricated from LH and HL sub businesses framed making use of DWT.

\subsection{Texture Feature Extraction}

Surface examination makes separation of typical and irregular tissue simple. It even gives differentiate among dangerous and ordinary tissue, which might be underneath the edge of human observation.. ground exam using computer helped willpower may be implemented to supplant biopsy techniques and assumes a big challenge in early give up and following of ailments. In first-request real ground research, statistics on ground is extricated from the histogram of image pressure. this technique estimates the recurrence of a specific dim degree at an unusual photograph role and may now not don't forget connections, or cooccurrences, amongst pixels. In 2nd-request true ground examination, data on ground is based upon on the possibility of finding more than one dark degrees aimlessly separations and guidelines over a whole picture. The actual highlights from MR pix are gotten utilising gray stage Cooccasion Matrix (GLCM), that is otherwise known as grey degree Spatial Dependence Matrix (GLSDM). GLCM, served with way way Haralick is real good approach can describe the spatial relationship between the pixels of severa dim stage.

The measurable ground highlights belief approximately are as in step with the following:

Complexity: Measures the community range within the dim degree cooccurrence grid, which may be determined as,

$$
\sum_{i, j}(i-j)^{2} P_{d, \theta}(i, j)
$$

Correlation: Measuring the degree of correlation pixel having a neighbor over the whole image. the range is [-1 1].

$$
\sum_{i, j} \frac{\left(i-\mu_{x}\right)\left(j-\mu_{y}\right) P_{d, \theta}(i, j)}{\sigma_{x} \sigma_{y}}
$$

Energy: Uniformity (or) Moment angle Secondly, returns the number of elements in a matrix of squares of the gray level co-occurrence. the range is $\left[\begin{array}{ll}0 & 1\end{array}\right]$.

$$
\sum_{i, j}\left(P_{d, \theta}(i, j)\right)^{2}
$$

Homogeneity: or moment Inverse Differential, returns a value that measures the level of closeness deprivation of components in the network dim dim co-event to the level of co-event network corner to corner. the range is [0 1] 


\section{GLCM of Fuzzy Clustering Means for Textural Future Extraction of Brain Tumor in Probabilistic Neural Networks}

$\sum_{i, j} \frac{p_{d, \theta}(i, j)}{1+|i-j|}$

Entropy: or Disorder

$$
\sum_{i, j} P_{d, \theta}(i, j) \log _{2}\left[\left(P_{d, \theta}(i, j)\right]\right.
$$

Where, $P_{d, \theta}(i, j)$ is the probability of finding a pixel with gray level $\mathrm{i}$ at a distance $\mathrm{d}$ and angle from a pixel with gray level $\mathrm{j}, \mu_{x^{\prime}} . \mu_{y}$ and $\sigma_{x}, \sigma_{y}$ are the mean and standard deviations of respectively. the ones measurable highlights can be furthermore sustained to the PNN classifier for making prepared and sorting out the presentation of the classifier in arranging the thoughts MR pics into everyday, favorable and threatening cerebrum tumors.

\section{RESULTS AND DISCUSSIONS}

In this examination, we have utilized two datasets, one was prepared dataset gathered from Web destinations www.diacom.com and the other was test dataset.. those datasets have been labored through the usage of the usage of skilled radiologists; this includes take a look at pics of 5 sufferers with all modalities. The records had been accumulated from automatic imaging and interchanges in medicinal drug dataset. we've had been given taken into consideration 650 amassed examples 25 pics of DICOM dataset, of which 18 are contaminated with brain tumor tissue and everyday for another exam. form judgments, directional highlights released from LL and HL subbands exchange offers wavelet ins and outs of the facts of the various bearings with more and more talk about the changes in the depiction of herbal tissue. Photographs MRI deteriorated into a beautiful 5 stages of the coefficient of the ins and outs of LL and HL subbands have been.

These subbands were gotten from wavelet disintegrated; the factual textural highlights, for example, vitality, relationship, entropy, and homogeneity were removed utilizing dim level co-event framework (GLCM).. Highlights texture obtained from severa phase wavelet damage have contemplated and has been achieved as a contribution of preparing and sorting the presentation of PNN classifier. Figure 1 to Figure 10 show various subbands ranges as well as a diploma fifth purchase wavelet decomposition. they highlight separated has been done as a vector to make the records furnished and check presentation of PNN classifier. Tables 1 and some of the views that attract the right texture, for example, relationships, differentiate, electricity, homogeneity and entropy given of darkish diploma co-activities lattice long-established at various levels LL and HL subbands each one of the five ranges organized and tried to photograph.

A lower estimate of the MSE and a higher estimate of PSNR shows the proportion of signal-tonoise better on the pictures pull out. Of perception, differences MRimages ready, at the same time because of the homogeneity of the MR image held snap turns are seen as much less at the same time as contrasted with pix MR trying. moreover, entropy and power determined in photographs MR held as identical as contrasted with photographs of MR attempt. With the proposed method and with the aid of real-time highlights texture (distinguishing, connection, power, homogeneity and entropy) secured from LL and HL subbands grouped photos into traditional mind tumors and anomalies. Differences in

the estimation of the real texture highlights of brain tumors organized and trying to look like was helpful in controlling the PNN classifier exhibition in getting geared up and try.

\begin{tabular}{llllll}
\hline Images & CON & COR & ENE & HOM & ENT \\
\hline Image 1 & 0.0116 & 0.0710 & 0.975 & 0.900 & 0.337 \\
Image 2 & 0.0112 & 0.0206 & 0.977 & 0.903 & 0.332 \\
Image 3 & 0.0036 & 0.0381 & 0.992 & 0.965 & 0.339 \\
Image 4 & 0.0139 & 0.0067 & 0.973 & 0.927 & 0.395 \\
Image 5 & 0.0168 & 0.0259 & 0.966 & 0.901 & 0.337 \\
Image 6 & 0.0054 & 0.0027 & 0.989 & 0.766 & 0.272 \\
Image 7 & 0.0138 & 0.0069 & 0.972 & 0.678 & 0.275 \\
Image 8 & 0.0047 & 0.0288 & 0.990 & 0.467 & 0.337 \\
Image 9 & 0.0162 & 0.0081 & 0.967 & 0.732 & 0.272 \\
Image 10 & 0.0125 & 0.0477 & 0.974 & 0.683 & 0.337 \\
\hline
\end{tabular}

Table 1 Features statistics obtained from the matrix of gray level co-occurrence (GLCM) of LL and HL subbands trained image

\begin{tabular}{llllll}
\hline Images & CON & COR & ENE & HOM & ENT \\
\hline Image 1 & 0.0098 & 0.0510 & 0.856 & 0.930 & 0.228 \\
Image 2 & 0.0073 & 0.0198 & 0.899 & 0.870 & 0.389 \\
Image 3 & 0.0110 & 0.0295 & 0.954 & 0.910 & 0.321 \\
Image 4 & 0.0095 & 0.0054 & 0.774 & 0.882 & 0.350 \\
Image 5 & 0.0120 & 0.0243 & 0.832 & 0.891 & 0.302 \\
Image 6 & 0.0043 & 0.0034 & 0.820 & 0.745 & 0.253 \\
Image 7 & 0.0100 & 0.0056 & 0.854 & 0.798 & 0.265 \\
Image 8 & 0.0030 & 0.0266 & 0.860 & 0.950 & 0.330 \\
Image 9 & 0.0130 & 0.0071 & 0.789 & 0.947 & 0.232 \\
Image 10 & 0.0108 & 0.0450 & 0.893 & 0.864 & 0.330 \\
\hline
\end{tabular}

Table 2 Features statistics obtained from the matrix of gray level co-occurrence (GLCM) LL and HL subbands tested image
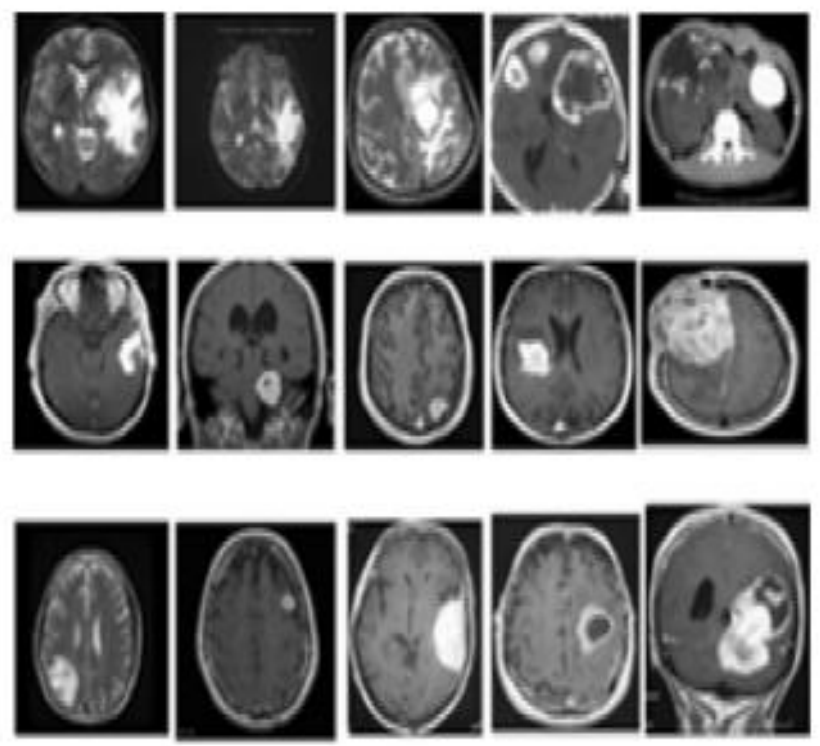

Fig.4 Brain tumor image dataset

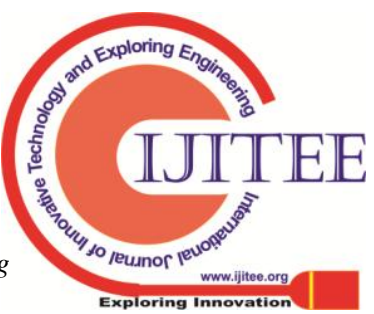


The belief outcomes are regarded in Fig. 6 talking to precise images (a) segment insightful, (b) preprocessed pics obtained via placing aside of clamor, (c) district based totally absolutely branch images, (d) removed tumorinfluenced locale from divided pix, vicinity of the tumorstimulated place.

5.1 Tumor identification precision examination by utilizing CNN( convolutional nueral systems)

The presentation of the $\mathrm{CNN}$ is estimated in each network layer. The objective of this progression is to recognize the ideal number of system layers for undertaking the most advanced precision and the briefest problem extraction time. The planned presentation of every layer is recorded in desk three. it is exhibited in table 3 that placing the quantity of layers equal to nine gave the fine execution. table 4 shows the nice precision were given from the three $\mathrm{CNN}$ fashions utilized in this research and the relating layer range. The machine duration has been more right to offer the remarkable execution in mastering diploma and in a while has been fixed, with the approval and attempting out degrees using a similar tool size because the reading diploma. this is to sum up the CNN version for all facts assessments.

\begin{tabular}{llll}
\hline \multirow{2}{*}{ Layer number } & \multicolumn{3}{l}{ Evaluation metrics } \\
\cline { 2 - 4 } & No. of features & Feature extraction time & Accuracy (96) \\
\hline Layer 2 (conv) & 290400 & 0.24 & 69.16 \\
Layer 3 (ReLU) & 290400 & 0.25 & 84.58 \\
Layer 4 (norm) & 290400 & 0.33 & 68.28 \\
Layer 5 (pool) & 69984 & 0.35 & 66.96 \\
Layer 6 (conv) & 186624 & 0.78 & 69.16 \\
Layer 7 (ReLU) & 186624 & 0.80 & 48.01 \\
Layer 8 (norm) & 186624 & 0.83 & 77.97 \\
Layer 9 (pool) & 43264 & 0.85 & 99.55 \\
Layer 10 (conv) & 64896 & 1.20 & 49.33 \\
Layer 11 (ReLU) & 64896 & 1.21 & 81.93 \\
Layer 12 (conv) & 64896 & 1.40 & 65.63 \\
Layer 13 (ReLU) & 64896 & 1.41 & 98.23 \\
Layer 14 (conv) & 43264 & 1.59 & 88.54 \\
Layer 15 (ReLU) & 43264 & 1.60 & 96.47 \\
\hline
\end{tabular}

Table 3 The amount obtained from the features, feature extraction time is consumed, and the accuracy of the different layers of $\mathrm{CNN}$

\begin{tabular}{lll}
\hline Network model & Layer number (type) & Accuracy (\%) \\
\hline AlexNet & Layer 9 (pool) & 99.55 \\
VGG-16 & Layer 32 (pool) & 92.95 \\
VGG-19 & Layer 38 (pool) & 95.15 \\
\hline
\end{tabular}

Table 4 Comparison of the three-phase model performance used in the detection of tumors $\mathrm{CNN}$

To further investigate the performance of classical learning model in the model-based against the proposed $\mathrm{CNN}$, classic brain tumor diagnosis method has been applied and brought to flower using a database design that are indistinguishable from in table 2. The proposed framework implemented $\mathrm{CNN}$ ninety nine.Fifty five\% accuracy, while the approach of an antique style finish 66.ninety six\%. A brief presentations correlation techniques vintage style deep past and current reading frame is proposed utilizing a comparable database added in table 5 . The consequences obtained in Table 5 shows the prevalence of depth to recognize the approach as a way of protracted as brain tumor identification accuracy, the association referred to in the MRI.

\begin{tabular}{|c|c|c|c|c|c|c|}
\hline \multirow{2}{*}{ Detection system } & \multicolumn{6}{|c|}{ Performance evaluation metrics (\%力) } \\
\hline & Sensitivity & Specticity & Accuracy & $B A$ & $P P V$ & $\overline{N P V}$ \\
\hline Abd-Ellahet al.(18) & 83.43 & 25.00 & 66.96 & 54.21 & 73.91 & 37.20 \\
\hline Proposed method & 99.38 & 1000 & 9955 & 99.69 & 100 & 9846 \\
\hline
\end{tabular}

Table 5 Comparison of the accuracy of detection of tumors derived from the $\mathrm{CNN}$-based system proposed in previous work where the conventional approach used by the same database as an integrated reference

Table 5 Comparison of the accuracy of the detection of tumor derived from the CNN-based system proposed in a previous work in which the conventional approach used by the same basis as the integrated reference exposure CNN provided in each layer of the device. The purpose of this development is to understand the proper amount of coating machines for precision sport out of the most extended and short extraction time element. Presentation of deliberately each layer is recorded in the table three. it exhibited in table 3 that put the same amount for the nine layers give the famous execution. four beautiful table shows the accuracy of the three modes has given $\mathrm{CNN}$ executed in this test and compare various broad layers. The length of the tool has been streamlined to offer superb execution in learning degrees and while there has been consistent, with the consent and try long stages utilize the same system as the learning rate. This is to summarize the model for all the exam notes CNN.
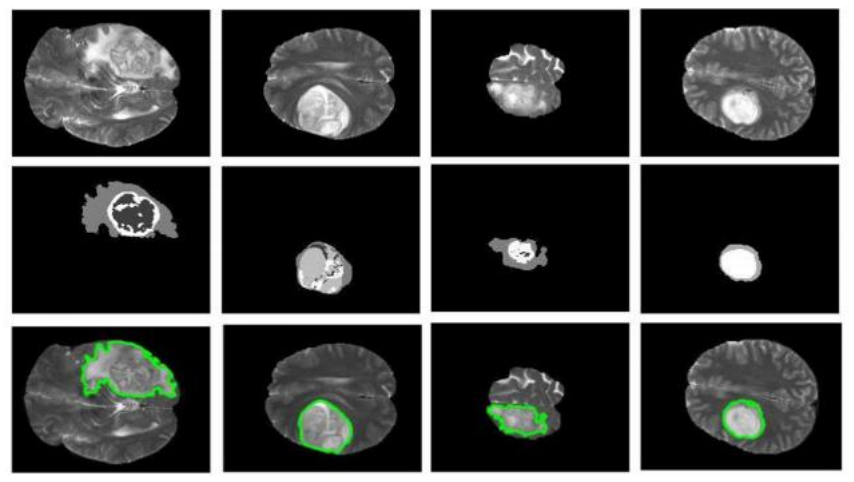

Fig.5 Visual results in axial view of the localization phase. The top row shows T2 modalities. The next line shows the ground truth. lowest column shows a fragmented picture

In elegant, the incredible execution is come to via using the input CascadeCNN model. It improves the cube diploma on all tumor locales. With this layout, we had the choice to attain.

Published By:

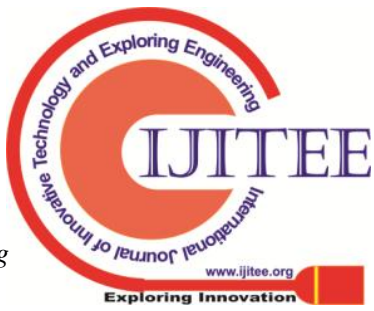



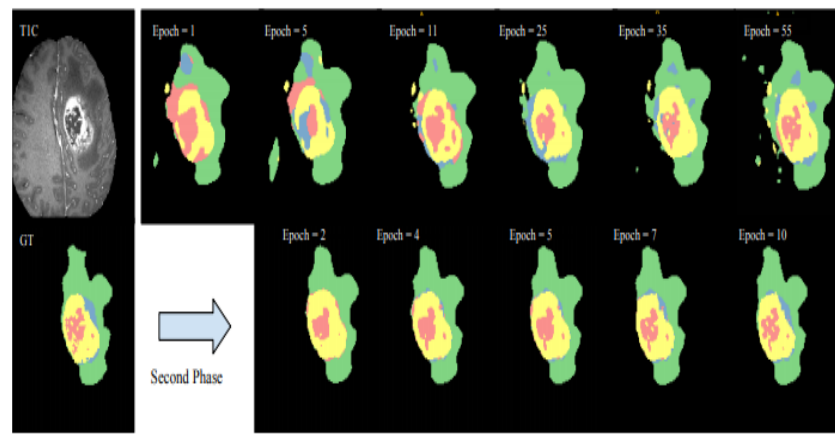

Figure 6: Progression of learning in InputCascadeCNN

\begin{tabular}{|c|c|c|c|c|c|c|c|c|c|}
\hline \multirow[t]{2}{*}{ Method } & \multicolumn{3}{|c|}{ Dice } & \multicolumn{3}{|c|}{ Specificity } & \multicolumn{3}{|c|}{ Sensitivity } \\
\hline & Complete & Core & Enhancing & Complete & Core & Enhancing & Complete & Core & Enhancing \\
\hline TwoPsтнCNN" & 0.85 & 0.78 & 0.73 & 0.93 & 0.80 & 0.72 & 0.80 & 0.76 & 0.75 \\
\hline Loca.PamiCNN* & 0.85 & 0.74 & 0.71 & 0.91 & 0.75 & 0.71 & 0.80 & 0.77 & 0.73 \\
\hline AverageCNN* & 0.84 & 0.75 & 0.70 & 0.95 & 0.83 & 0.73 & 0.77 & 0.74 & 0.73 \\
\hline GLовм.PaтнCNN* & 0.82 & 0.73 & 0.68 & 0.93 & 0.81 & 0.70 & 0.75 & 0.65 & 0.70 \\
\hline TwoPAтиCNN & 0.78 & 0.63 & 0.68 & 0.67 & 0.50 & 0.59 & 0.96 & 0.89 & 0.82 \\
\hline Locн.PaтиCNN & 0.77 & 0.64 & 0.68 & 0.65 & 0.52 & 0.60 & 0.96 & 0.87 & 0.80 \\
\hline
\end{tabular}

Table 6: Performance Two PathCNN models and variations

\section{PERFORMANCE ANALYSIS}

Images are trained dataset where the extracted features are trained using a probabilistic neural network (PNN) classifier for classification purposes, whereas disorganized test dataset using PNN classifier,definitely the real highlights and texture has been pulled out. The accuracy of photos organized and try to be around the concept of relying on tumor tissue associations unusual and strange. decided 7 shows the accuracy that carries around a grouping of tumor tissue and outstanding. Precision or right tempo characterization is skillability association in accordance with all checks out quantity orders. This approach of mind tumor characterization has been completed on one-of-a-kind daily MR and weird pix and controlled PNN classifier accuracy, usage scenarios given below

$$
\text { Accuracy }(\%)=\frac{\text { Correct cases }}{\text { Total number }} \times 100
$$

\begin{tabular}{llllc}
\hline Images & PSNR & MSE & Area of image in pixel & Area of tumor region \\
\hline Image 1 & 14.011 & 6.121 & 39,240 & 7698 \\
Image 2 & 13.82 & 3.116 & 67,824 & 9874 \\
Image 3 & 14.12 & 8.068 & 50,508 & 7423 \\
Image 4 & 13.86 & 4.77 & 50,388 & 9056 \\
Image 5 & 13.79 & 5.84 & 24,964 & 4564 \\
Image 6 & 13.82 & 7.79 & 50,429 & 3698 \\
Image 7 & 13.99 & 6.92 & 50,298 & 5879 \\
Image 8 & 14.004 & 7.35 & 35,040 & 13,923 \\
Image 9 & 14.066 & 6.215 & 50,544 & 6534 \\
Image 10 & 14.03 & 6.172 & 16,384 & 4497
\end{tabular}

Table 7 Evaluation of performance and area calculation extracted tumor trained image region
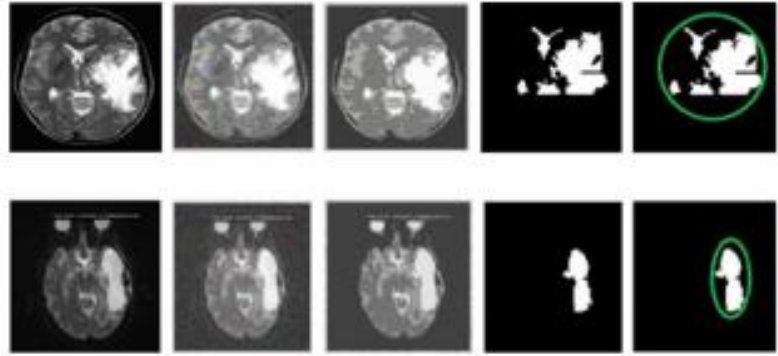

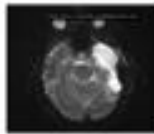

(a)

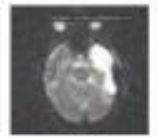

(b)

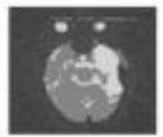

(c)

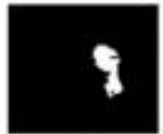

(d)

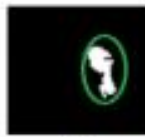

(e)
Fig.7 The observation of an image of the original image, the image preprocessed $b$, $c$ tumor image segmentation region, $d$ images extracted tumor, extracted the tumor area e area

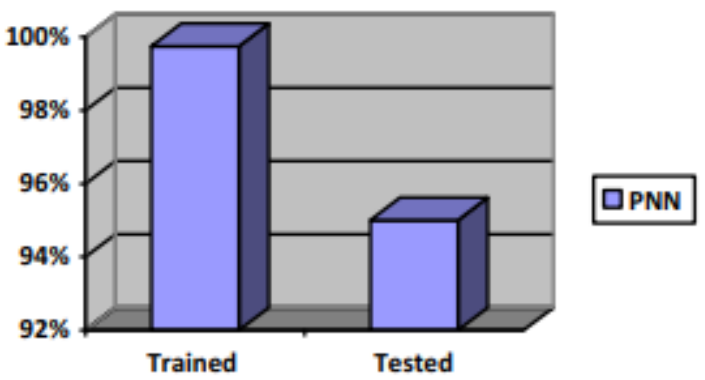

Fig. 8 Comparison of classification dataset trained and tested using probabilistic neural network

In the identification and classification of tumors of normal and abnormal brain MR images, the accuracy of almost $100 \%$ come to do for datasets prepared in the small fact that highlights the texture of measurable released from LL and HL subbands wavelet decomposition and $905 \%$, which was executed for attempting dataset. With a consequence of the above, we consider that the proposed method we unmistakably recognize the tumor where the maid everyday and normal in making the selection through the net assessment of medical experts.

\section{CONCLUSION}

This paper reward efficient method of classifying brain MR images into a normal tumors, benign and malignant, using a probabilistic neural network. , The proposed technique yielded promising results in MR photo books. Most contemporary strategies can recognize only a day and MR images into the mind wellknown and rarely [2]. At the same time, the proposed method, with the help of ground measurements has been given on the LH and HL sub companies, can order a brain tumor in the liver and dangerous. Precision volume of orders PNN use is seen as close to $100 \%$, when ready for a decent spread. Given the

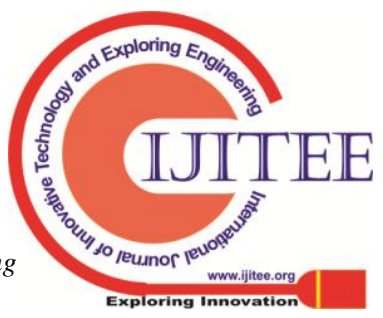


consequences exploration, PNN considered to have a broad focal factor above normal nervous system, because of the way that the income of the facts PNN quick education. Provide analysis speed PNN-solving ability to analyze a class by class. This technique for the identification and characterization of MR images programmed early in the normal mind, wise and dangerous, given their measurable peak surface, replacing the daily system clearly, but permits further reducing the cost of the victim.

\section{FUTURE SCOPE}

future work will be dealing with a brain tumor classification into different classes by using sophisticated texture analysis methods, so that the result of the cessation of brain tumors can be extended.

\section{REFERENCES}

1. Bahadure NB, Ray AK, Thethi HP (2017) photograph exam for MRI primarily based mind tumor place and highlight extraction using organically encouraged BWT and SVM. Int J Biomed Imaging 2017, Article identification 9749108, 12 pages

2. Joseph RP, Singh CS, Manikandan M (2014) thoughts tumor MRI picture department and recognition in photo getting prepared. Int J Res EngTechnol 3, eISSN: 2319-1163, pISSN: 2321-7308

3. Alfonse M, Salem M (2016) A programmed order of cerebrum tumors via MRI the use of bolster vector device. Egypt ComputSci J 40:11-21

4. Coatrieux G, Huang H, Shu H, Luo L, Roux C (2013) A watermarking-based totally completely medicinal picture honesty manage framework and a photo minute mark for converting portrayal. IEEE $\mathbf{J}$ Biomed health inform 17(6):1057-1067

5. Zanaty EA (2012) willpower of darkish problem (GM) and white hassle (WM) quantity in cerebrum attractive reverberation pics(MRI). Int J ComputAppl 45:16-22

6. Yao J, Chen J, Chow C (2009) Breast tumor exam mainly difference upgraded MRI utilising floor highlights and wavelet exchange. IEEE $\mathbf{J}$ Sel pinnacle signal system 3(1):ninety 4-one hundred

7. Kumar P, Vijayakumar B (2015) thoughts tumor MR image department and affiliation utilizing by way of manner of using PCA and RBF bit based totally completely help vector device. center East J Sci Res 23(nine):2106-2116

8. Sharma N, Ray A, Sharma S, Shukla adequate, Pradhan S, Aggarwal L (2008) Segmentation and characterization of recuperation snap shots using ground crude highlights: usage of BAM-kind counterfeit neural device. J Med Phys 33(3):119-126

9. Cui W, Wang Y, Fan Y, Feng Y, Lei T (2013) Localized FCM grouping with spatial information for restorative photo division and predisposition area estimation. Int $\mathrm{J}$ Biomed Imaging 2013, Article identity 930301,eight pages

10. Chaddad A (2015) computerized detail extraction in mind tumor with the aid of attractive reverberation imaging the use of Gaussian combination fashions. Int $\mathrm{J}$ Biomed Imaging 2015, Article ID868031, 11 pages 11. Sachdeva J, Kumar V, Gupta I, Khandelwal N, Ahuja CK (2013) Segmentation, highlight extraction, and multi beauty thoughts tumor order. J Digit Imaging 26(6):1141-1150

11. KailashD.Kharat, PradyumnaP.Kulkarni and M.B.Nagori "mind Tumor magnificence using Neural network primarily based completely definitely strategies" global magazine ofComputerScienceandInformatics, Vol-1, no.4, 2012

12. PankajSapra, Rupinderpal Singh and ShivaniKhurana , "thoughts Tumor Detection the usage of Neural community" ,worldwide JournalofScienceandModern Engineering, vol.1, no.9, 2013.

13. A.P. Nanthagopal and R.Sukanesh, "Wavelet measurable floor highlights based absolutely branch and order of thoughts figured tomography images", picture Processing, IET, vol.7, no. 1 pp. 25 - 32, 2013 [20] .

14. Shaik Salma Begum, Dr.D.Rajya Lakshmi "becoming a member of Wavelet actual floor and intermittent neural device for tumor identity and order over MRI, international mag of Engineering and superior technology (IJEAT) ISSN: $2249-$ 8958, quantity-8, hassle-6, August 2019. internet page no: 3769-3778. DOI : 10.35940/ijeat.F9388.088619 